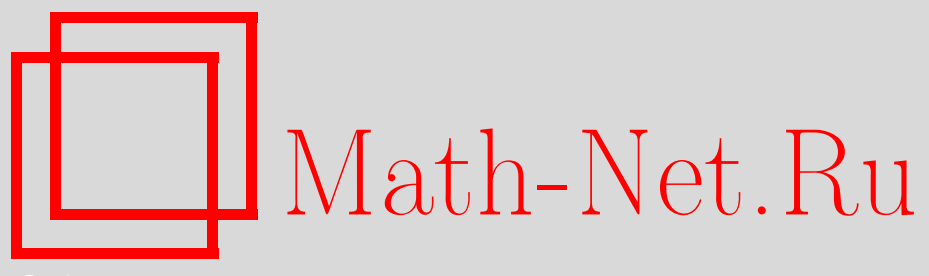

P. Р. Парвани, Некоторые соображения по поводу теоретико-информационного обоснования нелинейного уравнения Шредингера, ТМФ, 2007, том 152, номер 1, 157-162

DOI: https://doi.org/10.4213/tmf6077

Использование Общероссийского математического портала Math-Net.Ru подразумевает, что вы прочитали и согласны с пользовательским соглашением http://www . mathnet.ru/rus/agreement

Параметры загрузки:

IP : 54.162 .127 .20

26 апреля 2023 г., 12:32:03

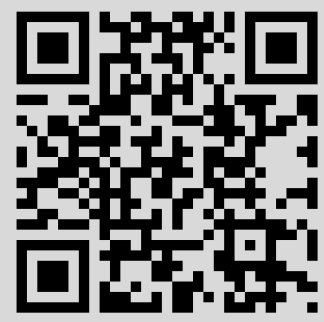




\title{
НЕКОТОРЫЕ СООБРАЖЕНИЯ ПО ПОВОДУ ТЕОРЕТИКО-ИНФОРМАЦИОННОГО ОБОСНОВАНИЯ НЕЛИНЕЙНОГО УРАВНЕНИЯ ШРЕДИНГЕРА
}

\begin{abstract}
Приведен обзор основных идей, приводящих к нелинейному обобщению уравнения Шредингера в контексте принципа максимальной неопределенности. Рассмотрены некоторые точные и пертурбативные свойства этого уравнения; соответствующие результаты зависят от свободного параметра регуляризации интерполяции $\eta$. Обсуждается вопрос о выборе этого параметра из энергетических соображений. Другими рассматриваемыми вопросами являются вопрос о таком выборе внешнего потенциала линейной теории, чтобы воспроизвести некоторые необычные точные решения нелинейного уравнения, и возможное расширение симметрии в нелинейной теории.
\end{abstract}

Ключевые слова: нелинейное уравнение Шредингера, теория информации, вырождение.

\section{1. ВВЕДЕНИЕ}

Одной из целей науки является построение экономичных теорий наблюдаемых явлений. На практике точность и диапазон имеющихся данных ограничены технологическими возможностями, временем, которое можно потратить на получение этих данных, и различными неконтролируемыми флуктуациями. В статистической механике и квантовой теории флуктуации описываются с использованием вероятностей, однако между этими двумя областями принято проводить различие в том смысле, что флуктуации внутренне присущи квантовой механике, в то время как в статистической физике они представляют собой удобный способ описания большой системы.

Понятие энтропии, или информации, сыграло ключевую роль в развитии статистической механики, а принцип максимальной энтропии (неопределенности) [1] является элегантным способом вывода описывающих систему распределений вероятностей, который, по существу, побуждает к беспристрастному выбору в рамках заданных ограничений, например при фиксированном значении энергии. Чтобы использовать этот метод, необходимо уметь измерять количество информации или,

${ }^{*}$ Department of Physics and University Scholars Programme, National University of Singapore, Kent Ridge, Singapore. E-mail: parwani@nus.edu.sg 
что эквивалентно, неопределенности. Шеннон построил свою меру неопределенности, потребовав, чтобы она удовлетворяла ряду правдоподобных аксиом [2]; изменение этих аксиом приводит к другим мерам неопределенности и, посредством метода максимальной неопределенности, к другим результатам для той же системы.

Оказалось, что нерелятивистское уравнение Шредингера может быть понято в рамках философии, основанной на принципе максимальной неопределенности [3]. Если начальное положение классической частицы не является известным точно, то принимается вероятностный подход, что приводит к динамике классического ансамбля. Максимизируя неопределенность путем минимизации соответствующей информационной меры Фишера, мы приходим к уравнению Шредингера.

Использование меры Фишера может быть оправдано на основе приемлемых в контексте динамики классического ансамбля аксиом [4], подобно тому как мера Шеннона была выведена из слегка отличающегося набора аксиом, подходящего для случая статистической механики. Нельзя утверждать, что две указанные информационные меры никак не связаны друг с другом; связь между ними может быть установлена посредством меры, называемой пространственной мерой Каллбэка-Лейблера (КЛ). Мера Фишера возникает из меры КЛ при обращении в нуль масштаба длины $L$, а мера Шеннона формально представляет собой предел меры КЛ при $L$, стремящемся к бесконечности [3], [5].

Таким образом, кажется естественным рассмотреть обобщенное (нелинейное) уравнение Шредингера, которое возникает из динамики классического ансамбля при использовании меры КЛ в качестве меры в методе максимальной неопределенности. Уравнение, получаемое в пространстве размерности больше единицы, не является инвариантным относительно вращений; это наводит на мысль о том, что пространственно-временнь́е симметрии могут быть связаны с квантовой линейностью, а величина $L$, возможно, связана с гравитационными эффектами [5].

Рассмотрим для простоты одну частицу в одномерном пространстве. Нелинейное уравнение имеет вид

$$
i \hbar \frac{\partial \psi}{\partial t}=-\frac{\hbar^{2}}{2 m} \frac{\partial^{2} \psi}{\partial x^{2}}+V(x) \psi+F(p) \psi
$$

где

$$
F(p)=\mathcal{E}\left[\ln \frac{p(x)}{p(x+L)}+1-\frac{p(x-L)}{p(x)}\right]+\frac{\hbar^{2}}{2 m} \frac{1}{\sqrt{p}} \frac{\partial^{2} \sqrt{p}}{\partial x^{2}}
$$

и $p(x)=\psi^{\star}(x) \psi(x)$. Масштаб длины нелинейности $L$ и энергетический параметр $\mathcal{E}$ связаны соотношением

$$
\mathcal{E} L^{2}=\frac{\hbar^{2}}{4 m},
$$

которое гарантирует, что ведущий член разложения уравнения (1) при малых значениях $L$ дает обычное линейное уравнение Шредингера.

Нелинейное уравнение Шредингера (1) напоминает дифференциально-разностное уравнение, поскольку в любой фиксированный момент времени эволюция волновой функции определяется не только информацией в точке $x$, но также и во всех соседних точках, находящихся от нее на конечном расстоянии, $x \pm L$. Нелинейность также 
является неполиномиальной. Тем не менее это уравнение имеет ряд важных общих свойств с линейным уравнением Шредингера таких, как сохранение вероятности и существование решений вида обычных плоских волн.

Приведенное выше уравнение следует регуляризовать, поскольку существуют потенциально сингулярные точки, в которых функция $p(x)$ обращается в нуль. Пусть

$$
p_{ \pm}(x) \equiv p(x \pm \eta L)
$$

где безразмерный параметр $\eta$ принимает значения в интервале $0<\eta \leqslant 1$. Тогда в правой части формулы (2) вместо первого члена нужно использовать следующее регуляризованное выражение:

$$
\frac{\mathcal{E}}{\eta^{4}}\left[\ln \frac{p}{(1-\eta) p+\eta p_{+}}+1-\frac{(1-\eta) p}{(1-\eta) p+\eta p_{+}}-\frac{\eta p_{-}}{(1-\eta) p_{-}+\eta p}\right]
$$

Формально параметр $\eta$ играет также роль интерполяционного параметра, поскольку при $\eta=1$ мы имеем полностью нелинейную теорию, а при $\eta=0$ - обычную линейную квантовую механику.

\section{2. ВОЗМУЩЕНИЯ И ЗНАЧЕНИЕ ПАРАМЕТРА $\eta$}

Уравнение Шредингера очень хорошо проверено, так что любая фундаментальная нелинейность должна быть очень малой. Поэтому при вычислении главных поправок к энергетическому спектру для различных внешних потенциалов нелинейность можно рассматривать как возмущение к обычному линейному уравнению Шредингера. В работах [6] было обнаружено, что в случае гладких внешних потенциалов $V(x)$ энергетический сдвиг для невозмущенных состояний с узлами дается относительно простым выражением

$$
\delta E \approx \frac{\hbar^{2}|L| \pi}{6 m} \sqrt{\eta(1-\eta)}(1-4 \eta) \sum_{p=1}^{N} C_{n p}^{2}+O\left(\frac{L}{a}\right)^{2},
$$

где $a$ - характерный масштаб длины линейной теории. Коэффициенты $C_{n p}$ зависят от наклона невозмущенных волновых функций вблизи узлов и, следовательно, от внешнего потенциала. Однако зависимость энергетических сдвигов от параметра $\eta$ является универсальной и выражается в положительных сдвигах энергии при малых значениях $\eta$ и отрицательных сдвигах при больших значениях.

До сих пор параметр $\eta$ являлся свободным параметром, $0<\eta<1$, описывающим семейство нелинейных уравнений. Можно, однако, рассматривать параметр $\eta$ как динамическую переменную в "пространстве теории" и фиксировать его, исходя из некоторых соображений. Одна из возможностей заключается в том, чтобы посмотреть, являются ли энергетические сдвиги минимальными для некоторого заданного значения $\eta$. Таким образом, мы предполагаем, что нелинейная теория движется в направлении тех значений $\eta$, которые минимизируют энергию системы. Элементарные алгебраические вычисления показывают, что обусловленные возмущением 
сдвиги, выражение для которых приведено выше, действительно достигают единственного глобального минимума при

$$
\eta_{\mathrm{m}}=\frac{7+\sqrt{33}}{16} \approx 0.80 .
$$

При этом значении параметра $\eta$ главные по порядку величины энергетические сдвиги являются отрицательными, так что нелинейность уменьшает энергию исходной линейной системы.

Результат (6) относится к случаю невозмущенных состояний с узлами. Для состояний без узлов имеем выражение [6]

$$
\begin{gathered}
\delta E(L) \propto L^{2} \eta^{2} \int_{-\infty}^{+\infty} \frac{d x}{p^{3}}\left[6(2-3 \eta)^{2}\left(p^{\prime}\right)^{4}-12\left(3-8 \eta+6 \eta^{2}\right) p\left(p^{\prime}\right)^{2} p^{\prime \prime}+\right. \\
\left.+4 p^{2} p^{\prime} p^{\prime \prime \prime}+p^{2}\left(3\left(p^{\prime \prime}\right)^{2}-2 p p^{\prime \prime \prime \prime}\right)\right],
\end{gathered}
$$

которое зависит от волновой функции и, следовательно, от потенциала. Заметим, что главный по порядку величины энергетический сдвиг для таких состояний без узлов подавляется дополнительным (по сравнению со случаем состояний с узлами) малым множителем $L / a$. Для основного состояния простого гармонического осциллятора имеем явное выражение

$$
\delta \widetilde{E}=\frac{\eta^{2}(1-\eta)(1-3 \eta)}{4}\left(\frac{L}{a}\right)^{2}+O\left(L^{4}\right) .
$$

Это выражение имеет единственный глобальный минимум при $\eta=(3+\sqrt{3}) / 6 \approx$ 0.79, что снова дает отрицательный вклад в энергию. Примечательно, что этот минимум весьма близок к универсальному минимуму для возбужденных состояний, $\eta_{\mathrm{m}} \approx 0.80$.

Если использовать волновую функцию основного состояния простого гармонического осциллятора в качестве вариационного приближения для основных состояний (без узлов) других внешних потенциалов, то можно прийти к правдоподобному заключению о том, что энергетические сдвиги основных состояний для всех гладких потенциалов даются приближенной формулой (9).

Это наводит на мысль о том, что $\eta_{\mathrm{m}} \simeq 0.8$ есть универсальное физическое значение параметра нелинейности, поскольку оно минимизирует энергию всех состояний в пространстве одного измерения.

Для систем с потенциалами, допускающими разделение переменных в трехмерном декартовом пространстве, таких, как частица в ящике или простой гармонический осциллятор, предыдущие результаты остаются в силе, поскольку трехмерная модификация уравнения (1) допускает разделение переменных [5].

В работах [6] указывалось, что при эвристическом использовании представленного выше квантово-механического результата в теории поля стандартные ультрафиолетовые расходимости квантовой теории поля могут оказаться ослабленными нелинейностью, которая может быть использована для эффективного описания гравитационных эффектов.

По поводу других исследований, касающихся нелинейных уравнений Шредингеpa, см., например, работы [7] и цитируемую там литературу. 


\section{3. ТОЧНЫЕ РЕШЕНИЯ И УСТОЙЧИВОСТЬ}

В работе [8] был построен класс точных решений для частицы на полупрямой $x>0$

$$
\psi_{e}(x, t)=C e^{-\kappa x} \alpha(x) e^{-i E t / \hbar},
$$

где $C$ - нормировочная константа, $\kappa>0$ и $\alpha(x)$ - периодическая функция, которая здесь выбрана равной

$$
\alpha(x)=\sin \left(\frac{2 \pi x}{\eta L}\right) .
$$

Собственное значение энергии $E$ дается выражением

$$
\left(1-\frac{E \eta^{4}}{\mathcal{E}}\right)=\ln [1+\eta(\gamma-1)]+\frac{1}{1+\eta(\gamma-1)},
$$

где

$$
\gamma \equiv e^{-2 \kappa \eta L}
$$

и ограничено как сверху, так и снизу при $0<\eta<1$ :

$$
0>E \geqslant \frac{\mathcal{E}}{\eta^{4}}\left(1-\ln (1-\eta)-\frac{1}{(1-\eta)}\right) .
$$

Нижняя граница является критической для энергетической устойчивости решения, однако, как видно, при $\eta \rightarrow 1$ ее значение неограниченно убывает.

Используя результат предыдущего раздела о том, что $\eta_{\mathrm{m}} \sim 0.8-$ физическое значение, получаем устойчивое невозмущенное (по $\eta$ ) решение нелинейного уравнения Шредингера. Это решение кажется весьма необычным: оно демонстрирует периодичность (экспоненциально затухающую) в отсутствие какого бы то ни было внешнего периодического потенциала. Более того, волновые функции в высшей степени вырожденны, поскольку можно выбрать любую функцию $\alpha(x \pm \eta L)=\alpha(x)$ и по-прежнему иметь то же собственное значение энергии.

\section{4. ЛИНЕЙНАЯ ТЕОРИЯ}

С учетом необычного поведения точного решения из последнего раздела было бы интересно рассмотреть вопрос о том, какой внешний потенциал позволяет воспроизвести подобное поведение в рамках линейного уравнения Шредингера. Таким потенциалом может быть потенциал

$$
V(x)=\left(\frac{E+\left(\hbar^{2} /(2 m)\right)\left(\partial^{2}\right)}{\psi_{e}}\right) \psi_{e},
$$

где функция $\psi_{e}$ задана формулой (10). Отсюда имеем

$$
V(x)=A+B \operatorname{ctg} \beta x,
$$

где $A, B, \beta$ суть параметры, связанные с параметрами в формуле (10). Сингулярности этого потенциала в точности совпадают с узлами волновой функции (10), так что линейное уравнение Шредингера в этом случае является хорошо определенным. Было бы интересно более широко исследовать этот периодический, но сингулярный потенциал.

6 Теоретическая и математическая физика, т. 152, № 1, 2007 г. 


\section{5. ОТКРЫТЫЕ ВОПРОСЫ}

Главная связанная с возмущением поправка для рассмотренных в разделе 2 возбужденных состояний обращается в нуль в трех точках: $\eta=0,1 / 4,1$. Для точки $\eta=0$ это не удивительно, поскольку эта точка формально представляет собой линейный предел. Факт обращения поправок в нуль в оставшихся двух точках представляется интригующим, поскольку в этих точках теория является нелинейной. Однако значение $\eta=1$ отвечает нерегуляризованной сингулярной теории, и, таким образом, только значение $\eta=1 / 4$ является, по-видимому, и осмысленным, и интересным. Поскольку связанная с возмущением энергетическая поправка для возбужденных состояний в этих точках обращается в нуль, возникает вопрос: не обнаруживает ли теория при этих значениях некоторой “защитной” симметрии?

Аналогично высокая степень вырождения точного решения из раздела 3 наводит на мысль о том, что теория может обладать некоторыми интересными скрытыми симметриями.

Многие модели единой теории в физике высоких энергий характеризуются введением дополнительных размерностей и спонтанным нарушением полной лоренцевой симметрии. Может ли нарушающая лоренцеву симметрию нелинейность, отмеченная в работе [5], быть связана с дополнительными размерностями? Несколько других подобных вопросов и предположений, связанных с рассмотренным нелинейным уравнением, обсуждаются в цитированной литературе.

Благодарности. Автор выражает благодарность Р. Конту, П. С. Део, С. Кару, Г. Табиа и Х.-С. Таню за плодотворные обсуждения по теме работы, а также организаторам конференции "Нелинейная физика: теория и эксперимент. IV" за теплое гостеприимство и возможность представить результаты в чудесной атмосфере Галлиполи.

\section{Список литературы}

[1] E. T. Jaynes, Probability Theory. The Logic of Science, Cambridge Univ. Press, Cambridge, 2003; R. Balian, Stud. Hist. Philos. Sci. B. Stud. Hist. Philos. Modern Phys., 36 (2005), 323.

[2] T. M. Cover, J.A. Thomas, Elements of Information Theory, Wiley Ser. Telecom., Wiley, New York, 1991.

[3] M. Reginatto, Phys. Rev. A, 58 (1998), 1775; Erratum 60 (1999), 1730; B. R. Frieden, J. Modern Opt., 35 (1988), 1297; Amer. J. Phys., 57 (1989), 1004.

[4] R. Parwani, J. Phys. A, 38 (2005), 6231; A physical axiomatic approach to Schrodinger's equation, quant-ph/0508125.

[5] R. Parwani, Ann. Physics, 315 (2005), 419.

[6] R. Parwani, G. Tabia, Universality in an information-theoretic motivated nonlinear Schrodinger equation, quant-ph/0607222.

[7] G. Svetlichny, Informal resource letter - nonlinear quantum mechanics on arXiv up to august 2004, quant-ph/0410036; R. Carroll, Remarks on the Schrodinger equation, quant-ph/0401082.

[8] R. Parwani, H. S. Tan, Exact solutions of a non-polynomially nonlinear Schrodinger equation, quant-ph/0605123. 